Cahiers de philosophie de l'université de

\title{
Les puissances subjectives de la nature selon Gabriel Tarde
}

Didier Debaise

\section{(2) OpenEdition}

12 Journals

Édition électronique

URL : https://journals.openedition.org/cpuc/325

DOI : 10.4000/cpuc.325

ISSN : 2677-6529

Éditeur

Presses universitaires de Caen

\section{Édition imprimée}

Date de publication : 31 décembre 2017

Pagination : 135-146

ISBN : 978-2-84133-859-7

ISSN : 1282-6545

\section{Référence électronique}

Didier Debaise, «Les puissances subjectives de la nature selon Gabriel Tarde », Cahiers de philosophie de l'université de Caen [En ligne], 54 | 2017, mis en ligne le 01 février 2019, consulté le 05 février 2023. URL : http://journals.openedition.org/cpuc/325 ; DOI : https://doi.org/10.4000/cpuc.325

\section{(c) (†) (8)}

Creative Commons - Attribution - Pas d'Utilisation Commerciale 4.0 International - CC BY-NC 4.0 https://creativecommons.org/licenses/by-nc/4.0/ 


\section{Les puissances subjectives de la nature selon Gabriel Tarde}

\section{Introduction}

Nous voulons proposer dans cet article une interprétation spéculative de la notion de sujet, à partir d'une reprise possible de la pensée de Tarde. Le projet se heurte de prime abord à d'innombrables difficultés et paradoxes: si la philosophie spéculative tente de soustraire les événements de la nature ${ }^{1}$ à toute inscription exclusivement anthropologique, humaine, n'est-il pas évident qu'elle ne peut le faire qu'en se dégageant de toute approche subjective de la nature? Et, si elle cherche effectivement à intégrer la notion de sujet à son enquête sur la nature, n'est-elle pas obligée de n'en faire qu'une dimension particulière, une phase ${ }^{2}$ ou une étape, dans un processus plus profond qui serait le devenir de la nature elle-même? Que pouvons-nous dès lors attendre d'une mise en relation entre deux termes qui ont cristallisé des tendances aussi opposées de la philosophie contemporaine? D’un côté le projet d'une anthropologie philosophique qui tente de penser la nature à partir de son inscription dans un sujet qui en fait effectivement l'expérience, de l'autre une philosophie de la nature qui se donne comme programme d'en dégager les caractéristiques sans les référer directement à un sujet anthropologique. L'alternative parait inévitable et toute tentative de mise en relation entre le sujet et la nature paraît devoir s'inscrire nécessairement dans l'une ou l'autre de ces voies.

1. Nous prenons ici «événement» au sens très large. Comme l'écrit G. Deleuze dans le chapitre du Pli qu'il consacre à la philosophie de Whitehead: «un événement, ce n'est pas seulement "un homme est écrasé": la grande pyramide est un événement, et sa durée pendant 1 heure, 30 minutes, 5 minutes..., un passage de la Nature, ou un passage de Dieu, une vue de Dieu» (G. Deleuze, Le pli: Leibniz et le baroque, Paris, Minuit, 1988, p. 103).

2. L'expression "phases d'individuation » est de G. Simondon. Notamment dans L'individuation psychique et collective, G. Simondon écrit: «L'unité et l'identité ne s'appliquent qu'à une des phases de l'être, postérieure à l'opération d'individuation » (G. Simondon, L'individuation psychique et collective: à la lumière des notions de forme, information, potentiel et métastabilité, Paris, Aubier, 1989, p. 14). 
Et si nous reprenions le problème différemment, en le sortant de l'alternative et en rétablissant un nouveau rapport, plus direct et plus constitutif. Si au lieu de les opposer nous faisions du sujet, non pas une phase ni un foyer d'expériences de la nature, mais sa réalité première, son point ultime d'existence; et si au lieu d'interpréter la nature comme un processus indifférencié et impersonnel, sorte d' «apeiron", "origine de toutes les espèces de l'être, antérieure à l'individuation " 3 , nous en faisions le résultat d'innombrables activités subjectives qui, à des échelles distinctes, se relieraient les unes aux autres et formeraient de véritables ensembles ou ordres de la nature; n'aurions-nous pas alors une tout autre vision des rapports entre le sujet et la nature? Nous en arriverions certainement à une étrange conception mais cependant pas si éloignée de celle imaginée notamment par William James lorsqu'il écrivait dans ses carnets qu'il s'agirait de penser un univers " de vies personnelles (qui peuvent être de niveaux de complexité différents, aussi bien suprahumaines, ou infrahumaines, qu'humaines), se connaissant les unes les autres par différents modes [...], évoluant et changeant véritablement par leurs efforts et leurs essais, et fabriquant le monde par leurs interactions et leurs succès cumulés ${ }^{4}$. On ne pouvait mieux dire quel concept de nature nous pourrions alors poser qui sortirait directement de l'alternative entre le sujet et la nature. Et si une telle approche renvoie à la philosophie spéculative, dont nous voudrions retracer ici les traits en reprenant celles de Tarde et de Whitehead, c'est que selon nous elle oblige à soustraire simultanément le concept de sujet de toute inscription anthropologique et le concept de nature de toute philosophie de la nature.

\section{Un nouvel espace de subjectivités}

Peut-on identifier des composantes minimales de la notion de sujet? Quel en serait le nombre et comment se relieraient-elles pour donner au sujet une consistance propre? Tarde et Whitehead voient dans le projet monadologique de Leibniz 5 , dont ils se veulent tous deux les héritiers, malgré de nombreuses

3. G. Simondon, L'individuation psychique et collective..., p. 196.

4. "[A universe $]$ of personal lives (which may be of any grade of complication, and superhuman or infrahuman as well as human), variously cognitive of each other [...], genuinely evolving and changing by effort and trial, and by their interaction and cumulative achievements making up the world» (W. James, Collected Essays and Reviews, Londres, Longmans, Green and Co., 1920, p. 443-444; nous traduisons en français).

5. Parce qu'elles se veulent toutes les deux des réactualisations complexes du projet monadologique, les philosophies de Tarde et de Whitehead peuvent être qualifiées de «néo-monadologiques», comme le sera aussi plus tard la philosophie de Raymond Ruyer (voir R. Ruyer, Néo-finalisme, Paris, PUF, 1952). 
réserves, l'origine d'une telle enquête sur les minima de subjectivité? Bien que le retour à Leibniz reste peu thématisé par eux lorsqu'il s'agit de la question du sujet, on peut néanmoins émettre l'hypothèse qu'ils furent fascinés par la définition que Leibniz donne dans La monadologie: un sujet est ce qui est capable de perceptions et d'appétitions ${ }^{6}$. On pourrait rajouter que, réciproquement, toute connexion entre des perceptions et des appétitions est le signe de l'existence d'un sujet. Le lexique leibnizien n'est pas exactement celui de la néo-monadologie: là où Leibniz parle de "perceptions", Tarde parle plutôt de "croyances" et Whitehead de "self-enjoyment", et là où Leibniz parle d' "appétition», Tarde utilise de préférence le terme «désir» et Whitehead celui de «but subjectif». Mais au-delà de la différence de lexique, la définition leibnizienne du sujet, pensé comme une connexion entre des perceptions et des appétitions, est reprise presque telle quelle dans l'approche néo-monadologique; c'est l'économie des relations existant entre les deux qui, comme nous le verrons, change entièrement. Dans tous les cas, la définition d'un sujet minimal (monade), pensé à l'intersection de deux forces, perceptive et appétitive, est le point de départ, l'impulsion, de la construction spéculative du sujet. On dira qu'il y a autant de sujets dans la nature qu'il y a de modes de perceptions et de types d'appétitions.

Que retenir, en première approximation, de cette inscription du sujet à l'intersection de ces deux forces et en quoi une telle inscription pourraitelle trouver une actualité dans la lecture spéculative que nous proposons? Tout d'abord, nous pouvons y voir une tentative importante pour sortir la question du sujet du paradigme de la conscience ou de l'aperception. En effet, ni la "perception» qui est définie comme un «état passager qui enveloppe et représente une multitude dans l'unité ou dans la substance

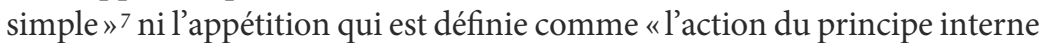
qui fait le changement ou le passage d'une perception à une autre ${ }^{8}$ ne renvoient à une quelconque faculté et encore moins à la conscience ou à l'«aperception $» 9$. La survalorisation de l'aperception a fait croire, comme l'écrit Leibniz, que «seuls les esprits étaient des monades et qu'il n'y avait point d'âmes des bêtes ni d'autres entéléchies ${ }^{10}$. En dégageant ainsi le sujet de l'aperception, Leibniz se donnait les moyens d'un élargissement considérable de son champ d'application dont il s'agirait de reprendre l'ambition. Ensuite, l'affirmation, déjà contenue dans la définition de la

6. G. W. Leibniz, La monadologie, Paris, Librairie générale française (Le livre de poche), 1991, p. 134.

7. Ibid., p. 130.

8. Ibid., p. 131.

9. Ibid., p. 130 .

10. Ibid. 
perception, selon laquelle les «monades vont toutes confusément à l'infini, au tout ${ }^{11}$ mais elles "sont limitées et distinguées par les degrés de perfections distinctes ${ }^{12}$, déplace complètement la question des limites et de l'identité du sujet, ou plus généralement de sa finitude. Comme l'écrit Tarde en écho à la pensée de Leibniz: "nul moyen de s'arrêter sur cette pente jusqu'à l'infinitésimal, qui devient, chose bien inattendue assurément, la clé de l'univers entier ${ }^{13}$. Le sujet devient infinitésimal, les petites perceptions qui le constituent vont à l'infini sous des modalités qui bien qu'elles soient de plus en plus imperceptibles n'en sont pas moins déterminantes. Ainsi, Tarde en fait le point central d'une redéfinition du sujet:

ce seraient donc les vrais agents, ces petits êtres dont nous disons qu' ils sont infinitésimaux, ce seraient les vraies actions, ces petites variations dont nous disons qu'elles sont infinitésimales ${ }^{14}$.

Ce changement d'échelle dans l'appréhension du sujet, que Tarde décrit comme le passage du fini à l'infinitésimal, et que Whitehead définit comme le passage du macroscopique au microscopique, a une conséquence majeure: le concept de sujet n'y est plus délimité à un domaine particulier de la nature. C'est à une telle extension de la notion que Whitehead en appelle dans Procès et réalité avec son concept d'entité actuelle qu'il définit aussi comme un sujet d'expérience:

Elles diffèrent entre elles: Dieu est une entité actuelle, et le souffle d'existence le plus insignifiant dans les profondeurs de l'espace vide en est une aussi. Mais, quoiqu'il y ait entre elles hiérarchie et diversité de fonction, cependant, dans les principes que manifeste leur actualisation, toutes sont au même niveau ${ }^{15}$.

Elles ne sont pas empiriquement ou pragmatiquement au même niveau, mais les principes dynamiques qui sont à l'œuvre dans des sujets tels que Dieu ou «le souffle d'existence le plus insignifiant » le sont. Ils sont à l'œuvre de la même manière, avec la même force, selon les mêmes réquisits. Ainsi les principes communs à tout sujet ne définissent pas des zones d'appartenance qui nous permettraient a priori de les hiérarchiser et de les distinguer par rapport à leur supposée adéquation à des principes fondateurs; ils n'ont pour prétention que de rendre importantes les dynamiques des sujets

11. G. W. Leibniz, La monadologie, p. 159.

12. Ibid.

13. G. Tarde, Monadologie et sociologie [1893], Paris, Institut d'édition Sanofi-Synthélabo (Les empêcheurs de penser en rond - Euvres de Gabriel Tarde, 1), 1999, p. 37.

14. Ibid., p. 40.

15. A. N. Whitehead, Procès et réalité. Essai de cosmologie, D. Janicaud (préface), Paris, Gallimard, 1995, p. 69. 
impliqués dans toute situation empirique. Il s'agit par là de mettre en correspondance un pluralisme radical, dont le principe est que le réel est constitué d'un "fourmillement d'individualités novatrices, chacune sui generis, marquée à son propre sceau distinct, reconnaissable entre mille ${ }^{16}$, et d'autre part une pensée de l'univocité affirmant «la discontinuité des éléments et l'homogénéité de leur être ${ }^{17}$.

\section{Les puissances de la possession}

Mais si nous disons avec Leibniz qu'un sujet se définit par son appétition, "qu'une spontanéité exacte nous est commune avec toutes les substances simples ${ }^{18}$, ce que Tarde reprend en disant qu'un sujet est une "action remarquable», et Whitehead qu'il est une entité actuelle ${ }^{19}$, la question principale est de savoir quelle est cette activité constitutive du sujet. Nous proposons de désigner cette activité constitutive, ou individuante, des sujets par un concept qui, bien qu'il n'occupe pas une place explicitement centrale ni chez Leibniz, ni chez Tarde ni même chez Whitehead, nous paraît cependant exprimer la spécificité du type d'approche des sujets qu'ils mettent en œuvre et des différences qui les animent. Ce terme générique désignant l'émergence des sujets de la nature, c'est la possession. Nous n'entendons par possession ni une forme de la propriété ${ }^{20}$ ni un mode de la domination d'un sujet sur un autre, mais le processus par lequel un sujet vient à l'existence.

Toute la philosophie s'est fondée jusqu'ici sur le verbe Être, dont la définition semblait la pierre philosophale à découvrir. On peut affirmer que, si elle eût été fondée sur le verbe Avoir, bien des débats stériles, bien des piétinements de l'esprit sur place auraient été évités. - De ce principe, je suis, impossible de déduire, malgré toute la subtilité du monde, nulle autre existence que la mienne; de là, la négation de la réalité extérieure ${ }^{21}$.

16. G. Tarde, Monadologie et sociologie, p. 65.

17. Ibid.

18. G. W. Leibniz, Essais de théodicée: sur la bonté de Dieu, la liberté de l'homme et l'origine du mal, J. Brunschwig (préface), Paris, Garnier-Flammarion, 1969, p. 291.

19. Toute la philosophie spéculative de Whitehead s'organise autour de cette notion d'entité actuelle: «Les "entités actuelles" - aussi appelées "occasions actuelles" - sont les choses réelles dernières dont le monde est constitué. Il n'est pas possible de trouver au-delà des entités actuelles quoi que ce soit de plus réel qu'elles» (A. N. Whitehead, Procès et réalité..., p. 68-69).

20. Sur la différence entre propriété et possession, nous renvoyons au texte d'I. Stengers: "William James: naturalisme et pragmatisme au fil de la question de la possession», in Philosophie des possessions, D. Debaise (dir.), Dijon, Les presses du réel, 2011, p. 35-69.

21. G. Tarde, Monadologie et sociologie, p. 86. 
Dans ses premiers écrits, Tarde s'exprime dans les termes d'une philosophie des possessions dont il reprend par la suite l'ambition par cette théorie de l'avoir. Ainsi, de la «nutrition dans le monde vivant», au "droit dans le monde social» en passant par «la perception dans le monde intellectuel», la philosophie de Tarde, dans ses premiers écrits, se présente comme une vaste enquête sur les modalités de la possession. Dans ses écrits ultérieurs, tels que Les lois de l'imitation et L'opposition universelle, il lui substitue d'autres catégories telles que l' «imitation ", l'«incitation ", la «subjugation", le «somnambulisme social» pour exprimer l'émergence et la consolidation de l'espace social, mais elles en gardent profondément la trace et pourraient être interprétées comme autant d'expressions de ce concept générique de la possession.

C'est toute une logique des sujets possessifs qu'il reste à construire sur la base de cette substitution de l'avoir à l'être à laquelle en appelle Tarde. Tout sujet impliquerait un certain type de questions concrètes: est-il engagé dans des possessions unilatérales ou réciproques? Dans quel rapport est-il l'acteur d'une possession et dans quel autre en est-il l'objet? Combien d'éléments - et avec quelle intensité - sont engagés dans ces rapports de possession? Tout sujet, selon Tarde, tend à une expansion maximale, utilisant d'innombrables moyens de capture afin de maintenir des alliances temporaires ou pour séduire en vue de maintenir sa domination sur les autres sujets. Les limites de son exercice sont toujours externes: «le rêve ambitieux d'aucune d'elles ne s'accomplit en entier, et les monades vassales emploient la monade suzeraine pendant que celles-ci $[s i c]$ les utilise ${ }^{22}$. La différence entre la «monade vassale» et la «monade suzeraine» n'est pas une différence de nature, mais une différence pragmatique: dans un milieu particulier un sujet est renforcé, par ses proximités avec d'autres, et devient capable d'une possession accrue. Car «elles font bien partie les unes des autres, mais elles peuvent s'appartenir plus ou moins ${ }^{23}$.

Dès lors, il n'y a aucune raison a priori de séparer les formes plus courantes de l'existence sociale, celles de collectifs engagés dans des relations de pouvoir, de résistance et de domination, de ce qui constitue chaque sujet. Nous aurions ainsi un théâtre microscopique de guerres, de conquêtes, de trahisons et de pacifications se jouant pour chaque sujet - un drame qui se démultiplierait à l'infini. Pourquoi les idées, les perceptions, les désirs, les croyances constitutifs d'un sujet n'exerceraient-ils pas eux aussi leurs conquêtes sur d'autres, ne s'allieraient-ils pas en vue d'accomplir une plus grande possession? Avoir une idée n'est-ce pas essentiellement être

22. G. Tarde, Monadologie et sociologie, p. 93.

23. Ibid. 
possédé simultanément par elle et une multiplicité d'autres qui lui sont associées pour un temps? Une croyance ne porte-t-elle pas directement avec elle d'autres croyances qui provisoirement lui sont soumises jusqu'au moment où, à l'occasion de l'introduction d'une nouvelle, les rapports se transforment? Pour Tarde, les désirs et les croyances, les inventions et les variations, sont eux aussi animés par ce grand principe: "tout être veut, non pas s'approprier aux autres êtres, mais se les approprier ${ }^{24}$.

C'est ici que la relation entre Tarde et Whitehead se complète particulièrement bien. Car, nous ne trouverons chez Tarde qu'une interprétation partielle de cette philosophie spéculative des possessions. Ce n'est pas là une critique mais la condition même des problèmes qu'il pose. L'intérêt de Tarde pour la philosophie spéculative est entièrement déterminé par l'ambition d'élargir le champ d'investigation de la sociologie en y introduisant des dimensions physiques, biologiques, éthologiques et humaines. Elle y représente un outil fondamental d'élargissement du champ d'investigation sur les manières de faire société. Elle n'est donc envisagée qu'à l'intérieur des situations toujours partiales de constitution de dynamiques collectives auxquelles elle vise à donner une plus grande amplitude. Le problème que pose Whitehead est quelque peu différent et lui permet de donner à la question de la philosophie spéculative une pertinence propre. Le soustitre de son principal ouvrage, Procès et réalité, fournit une indication fondamentale sur le statut des propositions qui sont mises en œuvre. Ce sous-titre est: Essai de cosmologie. Lorsque Whitehead parle d'expériences, de sujets, de sensations, de buts subjectifs, etc., nous devons toujours garder à l'esprit le fait qu'il s'agit de propositions essentiellement cosmologiques. Lorsqu'il utilise, comme Tarde, le concept de sujet, c'est d'un point de vue cosmologique et c'est pourquoi la question de la possession, comme constitutive de l'émergence d'un sujet de la nature, trouve chez Whitehead une dimension nouvelle, dégagée de toute situation empirique. L'exprimant dans le cadre d'une opposition à Kant, Whitehead écrit:

Pour Kant le procès par lequel il y a de l'expérience, est un procès de la subjectivité vers l'objectivité apparente. La philosophie de l'organisme inverse cette analyse et explique le procès comme procédant de l'objectivité vers la subjectivité, à savoir de l'objectivité par quoi le monde extérieur est un donné, vers la subjectivité, c'est-à-dire ce par quoi il existe une expérience individuelle ${ }^{25}$.

La validité ou non de la critique n'est pas très importante ici mais la question qu'elle exprime l'est particulièrement. En effet, ce qui est

24. Ibid., p. 89

25. A. N. Whitehead, Procès et réalité..., p. 264. 
intéressant n'est pas de savoir comment, et à quelles conditions, un sujet peut faire l'expérience du monde, mais au contraire, question beaucoup plus singulière, comment le monde devient subjectif. C'est donc, ce que nous proposons d'appeler, dans un vocabulaire différent de celui de Whitehead, la possession, qui devient le terme central de cette émergence du sujet dans et par le monde. C'est elle qui exprime le principe métaphysique que Whitehead place au centre de sa philosophie: "la pluralité, qui est l'univers pris en disjonction, devient l'occasion actuelle unique [sujet], qui est l'univers pris en conjonction ${ }^{26}$, ou encore «le principe métaphysique ultime est l'avancée vers la conjonction à partir de la disjonction, créant une entité nouvelle autre que les entités données en disjonction ${ }^{27}$.

La possession peut être donc pensée comme l'opération interne au monde par laquelle la pluralité de ce qui existe produit, ou devient, une unité d'expérience, un véritable sujet. Celui-ci n'est pas une synthèse du monde mais une prise ou une contraction. Le terme que Whitehead utilise pour désigner cette activité possessive est le terme de "préhension ${ }^{28}$. Tout se passe comme si l'univers ne cessait de se contracter en une multiplicité de points qui sont autant de centres d'expérience, de perspectives sur l'ensemble de ce qui existe. À chaque fois, c'est un point de perspective, non pas sur mais de la nature, un devenir subjectif, qui est en même temps un point de possession. Dans la lecture qu'il propose de la théorie de l'événement chez Whitehead, Deleuze en donne une série d'exemples:

Toute chose préhende ses antécédents et ses concomitants et, de proche en proche, préhende le monde. L'œil est une préhension de la lumière. Les vivants préhendent l'eau, la terre, le carbone et les sels. La pyramide à tel moment préhende les soldats de Bonaparte (quarante siècles vous contemplent), et réciproquement ${ }^{29}$.

L'influence de la pensée de la préhension chez Whitehead sur la philosophie de Deleuze ne se trouve pas uniquement dans Le pli; on la retrouve dans une filiation néo-platonicienne qui s'exprime notamment dans Différence et répétition. La préhension y apparaît comme une des formes de la «contraction» et de la «contemplation» plotinienne:

26. A. N. Whitehead, Procès et réalité..., p. 72.

27. Ibid., p. 73 .

28. Whitehead n’hésite pas à lier le concept aux Méditations de Descartes: «En langage cartésien, l'essence d'une entité actuelle consiste seulement en ce qu'elle est une chose qui préhende (c'est-à-dire "une substance dont toute l'essence ou la nature n'est que de préhender")» (ibid., p. 100).

29. G. Deleuze, Le pli: Leibniz et le baroque, p. 106. 
Le lys des champs, par sa seule existence, chante la gloire des cieux, des déesses et des dieux, c'est-à-dire des éléments qu'il contemple en contractant. Quel organisme n'est pas fait d'éléments et de cas de répétition, d'eau, d'azote, de carbone, de chlorures, de sulfates contemplés et contractés, entrelaçant ainsi toutes les habitudes par lesquelles il se compose? Les organismes s'éveillent sous les paroles sublimes de la troisième Ennéade: tout est contemplation ${ }^{30}$.

C'est ici qu'une distinction importante doit être établie entre la monadologie et la néo-monadologie. Si le projet leibnizien peut légitimement être décrit comme le point d'impulsion d'une théorie spéculative des sujets, qui trouvera chez Tarde et Whitehead ses outils principaux, cela ne réduit en rien la rupture nécessaire existant avec le projet initial. On pourrait la résumer en disant qu'à une logique de l'expression, il s'agirait d'opposer une logique de la possession. Quelle est la critique qu'ils peuvent adresser à la théorie de l'expression et qui constitue une des conditions principales d'une réactualisation du projet monadologique? On dira que dans la logique de l'expression «le monde, comme exprimé commun de toutes les monades, préexiste à ses expressions ${ }^{31}$. Certainement, l'univers «n'existe pas hors de ce qui l'exprime, hors des monades ellesmêmes; mais ces expressions renvoient à l'exprimé comme au réquisit de leur constitution ${ }^{32}$. Ce qui fonde la théorie de l'expression, c'est le présupposé d'un monde commun, démultiplié, comme l'écrit Leibniz, par ses perspectives, mais néanmoins unique. Avec la néo-monadologie, c’est l'univers lui-même qui se démultiplie, comme il se contracte, chaque sujet produisant un univers propre par l'opération de possession des autres. C'est littéralement un "déferlement de séries divergentes », l'irruption d' «incompossibilités sur la même scène, là où Sextus viole et ne viole pas Lucrèce, où César franchit et ne franchit pas le Rubicon, où Fang tue, est tué et ne tue pas ni n'est tué ${ }^{33}$. Chaque sujet est le centre d'émergence d'un nouvel univers qui est cependant formé des mêmes matériaux, mais cette fois, à la différence de Leibniz, ce matériau commun n'est pas l'univers exprimé, prédonné, mais uniquement les autres sujets capturés, devenus objets dans cette nouvelle relation. La question n'est plus de savoir comment un univers commun s'actualise dans une multiplicité de sujets mais à l'inverse de savoir comment cette multiplicité peut produire un ou plusieurs univers communs.

30. G. Deleuze, Différence et répétition, Paris, PUF, 1968, p. 102.

31. Ibid., p. 68.

32. Ibid.

33. G. Deleuze, Le pli: Leibniz et le baroque, p. 112. 


\section{Conclusion}

Nous nous sommes fixés comme objectif de sortir de l'opposition entre la nature et le sujet, imposant une oscillation permanente entre l'anthropologie philosophique et la philosophie de la nature. Une possibilité selon nous passe par une refonte spéculative de la notion de sujet de telle sorte qu'elle puisse devenir le premier terme d'une investigation sur la nature. Mais en nous focalisant autant sur les composantes du concept de sujet n'avons-nous pas fait trop rapidement l'économie de la nature elle-même? Si le sujet doit acquérir une extension aussi large dans l'interprétation spéculative, qu'il puisse devenir le terme de tout centre d'expérience animant des réalités aussi bien biologiques que psychologiques ou sociales, cela n'implique-t-il pas une disparition du concept de nature? En un mot: quelle place accorderons-nous à la nature? Qu'est-elle à présent censé désigner? Devons-nous introduire d'autres dimensions de l'existence dont nous aurions dû faire jusqu'à présent l'économie au risque de revenir à une différence entre le sujet et la nature?

La sortie de l'alternative peut s'exprimer par une phrase de Whitehead, de prime abord énigmatique, mais à laquelle il conviendrait de donner le statut d'un principe central de la philosophie spéculative: «mise à part l'expérience des sujets, il n'y a rien, rien, rien que le rien ${ }^{34}$. Dans la mesure où elle s'inscrit dans le cadre d'une pensée spéculative, elle est une prise de position forte sur l'existence en général. Elle signifie littéralement qu'il n'y a rien, ni nature, ni substance, ni être, ni chose, mais uniquement des sujets qui se lient les uns aux autres, aspirent, selon les mots de Tarde «au plus haut degré de possession; de là leur concentration graduelle» ${ }^{35}$. Des sujets qui en possèdent d'autres et sont possédés en retour, formant de véritables dynamiques d'existence collective que Tarde et Whitehead désignent par le même concept: des sociétés. Et lorsqu'ils utilisent le terme «société", c'est pour des réalités aussi différentes que l'existence d' "un objet physique ordinaire, qui a une durée temporelle ${ }^{36}$, la «vie d'un homme», "la grande pyramide d'Égypte», ou encore les «lois de la nature». À chaque niveau, physique, biologique ou social, nous trouvons des modes de la possession, une société se définissant comme «la possession réciproque, sous des formes extrêmement variées, de tous par chacun ${ }^{37}$. Il n'y a rien d'autre que les actions des sujets, ni substance collective, ni forme commune, ni fins

34. A. N. Whitehead, Procès et réalité..., p. 281.

35. G. Tarde, Monadologie et sociologie, p. 93.

36. A. N. Whitehead, Procès et réalité..., p. 91.

37. G. Tarde, Monadologie et sociologie, p. 85. 
générales auxquelles tendraient tous les membres d'une société déterminée. Nous n'avons pour rendre compte de la nature que des sujets et, sous des formes extrêmement variées, les activités possessives. C'est alors à une tout autre forme d'enquête que la philosophie spéculative en appelle, une enquête dans laquelle les composantes spéculatives et abstraites des sujets se trouvent engagées à l'intérieur des formes multiples de la possession dont le programme a été annoncé notamment par Tarde:

Depuis des milliers d'années, on catalogue les diverses manières d'être, les divers degrés de l'être, et l'on n'a jamais eu l'idée de classer les diverses espèces, les divers degrés de la possession ${ }^{38}$.

Didier Debaise

Fonds national de la recherche scientifique

Université libre de Bruxelles

38. Ibid., p. 89. 
